\title{
Esprits sexués et maux sexuels
}

\author{
Ce qu'il est bon de dire chez les \\ devins-guérisseurs malgaches
}

Olivia Legrip-Randriambelo ${ }^{1}$

\begin{abstract}
[Résumé] Cet article propose une relecture de discours recueillis auprès de guérisseurs et de croyants, portant sur la religion à la lumière de la sexualité, peu évoquée à Madagascar (qu'elle soit factuelle ou symbolique, dans le cas d'alliance maritale Homme/entité). L'enquête de terrain permet de saisir les articulations entre religion et sexualité dans les salles de soins des devins-guérisseurs et montre comment les devins-guérisseurs, les croyants et/ou les malades rencontrés sur ce terrain de recherche mené dans la région betsileo, au Sud des Hautes Terres centrales de lî̀le, composent leurs discours sur la sexualité en contexte religieux et face à l'ethnologue. La sexualité rencontre le religieux à la fois par le biais d'alliances ou de filiation, comme cause de la consultation, ou encore, peut être passée sous silence, voire détournée par la plaisanterie.
\end{abstract}

Mots-clés : religion, sexualité, alliance symbolique, discours, Madagascar.

[Abstract] This article proposes a rereading of discourses collected from healers and believers, regarding religion in light of sexuality, which is seldom evoked in Madagascar (be it factual or symbolic in the case of Man/entity marital alliances). The fieldwork studies the relationship between religion and sexuality in the care rooms of healersdiviners, in other words, it is about grasping how the healers-diviners and/or the patients encountered on this fieldwork conducted in the betsileo region, south of the Central Highlands of the island, compose their discourses on sexuality in a religious context and in front of the ethnologist. Sexuality meets religion either through alliances or filiation, as the cause of the consultation, is not mentioned or is deflected using humor.

Keywords: religion, sexuality, symbolic alliance, discourses, Madagascar.

\section{Introduction}

Les données ethnographiques que je propose de mettre en lumière ici m’ont imposé une relecture par le biais de la religion, et surtout, de la sexualité, peu évoquée par mes interlocuteurs malgaches (qu'elle soit factuelle ou symbolique, dans le cas d'alliance maritale Homme/entité) et, en général, dans les études anthropologiques menées dans l'océan indien occidental (Rakotomalala, 2010a). Les dimensions

\footnotetext{
${ }^{1}$ Université Lyon 2.
} 
sexuelles sont toutefois présentes dans les poésies malgaches : filan'ampela pour la région sakalava (Nord-Ouest de l'île) souvent érotiques et hain-teny pour la région merina (Nord des Hautes Terres, autour de la capitale Tananarive), même si leur symbolisme n'est pas toujours apparent (Hébert, 1964).

Ce texte interrogera les articulations entre religion et sexualité dans les salles de soins des devins-guérisseurs au prisme de l'enquête de terrain. Autrement dit, il s'agira de saisir comment les devins-guérisseurs, les croyants et/ou les malades rencontrés dans la région betsileo, au Sud des Hautes Terres centrales de lîle, composent leurs discours sur la sexualité en contexte religieux et face à l'ethnologue. La sexualité rencontre le religieux à la fois par le biais d'alliances ou de filiations, comme cause de la consultation, ou encore, peut être passée sous silence, voire détournée par la plaisanterie. Pour ce faire, je vais m'appuyer sur des cas ethnographiques recueillis entre 2007 et 2014.

\section{Rencontres avec les « filles-des-eaux »}

\subsection{Alliances avec des entités aquatiques}

Dans les discours et les représentations populaires, les entités aquatiques évoquent directement la féminité et la fertilité. Elles contractent régulièrement des mariages avec des hommes (pas seulement avec des devins-guérisseurs), ainsi par la filiation, les «filles-des-eaux " (zazavavindrano) ou "princesses-des-eaux » (andriambavirano) peuvent donner naissance à des lignages à l'apparence humaine. Des récits de contact avec les entités aquatiques et des légendes généalogiques ont été relevés dès les premiers écrits sur Madagascar (Flacourt, 2007 [1658] ; Dubois, 1938). En 1658, Étienne de Flacourt évoque « une femme marine qui accoucha sur le bord du sable [et] venait de temps en temps visiter son fils [...], dont les descendants sont encore vivants » au Sud de l'île (Terramorsi, 2010 : 14) ; ou encore, au début du XXe, siècle, en région betsileo, Henri Dubois (1938) décrit l'histoire d'un frère et d'une sœur tous deux engloutis dans des eaux douces après une rencontre avec des andriambavirano. Les alliances avec à des esprits des eaux ou de la forêt donnant naissance à des enfants au « destin singulier [...] de plain-pied dans les champs du religieux » (Saladin d'Anglure, Morin, 1998: 53) ont été remarqué dans de nombreux travaux ethnologiques, dont ceux de Bernard Saladin d'Anglure et Françoise Morin au Pérou et dans l'Arctique canadien, de Roberte Hamayon en Sibérie (1990) ou encore de Philippe Descola en Équateur (1993).

Dans le cas malgache, les humains ayant la réputation d'avoir entretenu des relations sexuelles et/ou maritales avec des esprits du monde invisible peuvent avoir une descendance visible. Ces humains sont alors des privilégiés, des intercesseurs ou des devins-guérisseurs dont les voyages subaquatiques ou les alliances sont l'aboutissement d'un processus initiatique. Tel que le souligne Roberte Hamayon c'est " comme si tous pouvaient "aimer" dans la surnature, mais certains seulement, "s'y 
marier” » (1990 : 436). Les filles-des-eaux malgaches sont un pont entre les divinités et les Hommes. Elles constituent donc un entre-deux performatif pour les Hommes à qui elles ouvrent l'accès au monde surnaturel. Par exemple, un de mes interlocuteurs, Donné, est guérisseur et responsable d'un lieu de culte situé au sommet d'une colline cernée d'une dense forêt où des esprits soignent dans la cavité obscure d'une grotte à proximité d'une source sacrée. Donné est le fils d'un homme et d'une andriambavirano, guérisseuse et résidente de la source sacrée, qui l'a porté durant deux mois avant le transfert magique qui a permis à l'épouse humaine de son père de mener à terme la grossesse, raconte-t-il. Cette filiation fait de Donné l'unique interlocuteur des entités présentes dans la grotte et la source sacrée située à proximité. Donné a, du fait de sa condition, effectué de nombreux voyages subaquatiques en diverses localités de l'île.

À la différence d'une guérisseuse vivant une possession amoureuse avec un esprit royal et héroïne d'un documentaire ethnographique réalisé dans l'ouest malgache (Fiéloux, Lombard, 1991), au cours de mon terrain la dimension amoureuse ou sexuelle a rarement été évoquée mais a plutôt été sous-entendue par mes interlocuteurs lors de l'évocation des «rencontres » et/ou des filiations. Elle a plutôt été présente dans le contenu des récits populaires et de l'imaginaire concernant les «fillesdes-eaux ».

\subsection{L'ethnologue et les esprits aquatiques}

Ces rencontres avec les filles-des-eaux ont par la suite été imbriquées à ma présence sur le terrain et me permettent de penser l'insertion de l'ethnologue dans le paysage religieux malgache. Il m’a été donné d'entendre, dans les salles de soin, une comparaison attestant de la proximité physique entre ces esprits de la nature et l'ethnologue vazaha (" étranger »). J'ai ainsi pu être rapprochée d’entités aquatiques, zazavavindrano (" filles-des-eaux ») ou andriambavirano (" princesses-des-eaux »), par plusieurs devins-guérisseurs ayant été formés auprès de ces dernières lors de voyages subaquatiques dans un court d'eau, à la suite de la révélation de leur don, afin d'acquérir leurs pleines connaissances magico-thérapeutiques. Lorsque je lui demandai de me décrire ces esprits aquatiques, RaJean-Pierre, guérisseur betsileo ayant passé sept jours en immersion, répond: «Les gens qui sont là-bas, il n’y a pas de différence avec toi. Ils sont blancs, comme les vazaha [" étrangers »]2.»

Après avoir noté ma ressemble physique (couleur de peau et cheveux) avec les zazavavindrano ("filles-des-eaux »), RaJean-Pierre a tenu à m'examiner à l'aide de son miroir divinatoire et en a conclu: «Toi, tu es le genre de personnes qui va aller dans

\footnotetext{
2 Traduction d'un extrait d'entretien réalisé le 6 juin 2011 à Ambalavato, Fianarantsoa.
} 
l'eau là-bas ${ }^{3}$ !», ce que son épouse Jeanne, invectivant son mari, a catégoriquement contredit, évoquant l'état d'ébriété de RaJean-Pierre ce matin-là :

R.-P. : Toi, tu es le genre de personnes qui va aller dans l'eau là-bas !

O. : Ha bon?

R.-P. : Je le sais tout de suite, hein !

J. : Est-ce que tu as quelque chose en toi ?

R.-P. : Ha, je vais t'amener hein!

J. : Aaa $^{4}$, ne l'amène pas ${ }^{5}$ !

Cette comparaison est un moyen de rendre à la fois concrets, réels et proche de moi, tant son activité que les entités qu'il fréquente régulièrement. Le guérisseur a ainsi développé son discours et entrepris d'évoquer sa seconde activité, celle qui consiste à guider auprès des andriambavirano ("princesses-des-eaux») les futurs devinsguérisseurs en formation qu'il prend en charge.

Le parallèle tracé avec moi n'est aucunement magique : il est purement physique. Les « filles-des-eaux» ont la peau blanche et des cheveux noirs bouclés, comme moi. Mes interlocuteurs intègrent ainsi au système magico-religieux local une personne qui en est a priori extérieure. Delphine Burguet (2012), ethnologue également comparée à une entité chtonienne malgache (kalanoro), voit dans ce rapprochement « le processus d'humanisation, une occidentalisation » (p. 196) de ces esprits. Ceci permettrait aux esprits de la nature souvent dépréciés par le système de pensée et de catégorisation chrétien, d'acquérir une meilleure légitimité, de même que l'ethnologue qui leur est associé. Il est alors possible de dispenser un discours magico-religieux qui n'est plus si éloigné du chercheur.

Ainsi, comme le soulignent Bernard Saladin d'Anglure et Françoise Morin (1998), le discours concret et réel tenu sur les esprits et les alliances faites avec eux les légitiment. Ils notent : « loin d'être perçus comme des parents métaphoriques par les sociétés qui s'y réfèrent, ils sont décrits comme faisant partie de la réalité autochtone, de l'environnement spirituel et humain, inséparables de l'environnement naturel » (p. 70).

\subsection{Alliances, filiations et sexualité déviante}

Comme les devins-guérisseurs, la grande majorité de mes interlocuteurs exorcistes laïcs (mpiandry) du mouvement de Réveil luthérien malgache (fifohazana) ${ }^{6}$ voient un

\footnotetext{
${ }^{3}$ Idem.

${ }^{4}$ L'interjection « aaa » exprime un « non » sans équivoque.

${ }^{5}$ Traduction d'un extrait d'entretien réalisé le 6 juin 2011 à Ambalavato, Fianarantsoa.

${ }^{6}$ Né en 1894 dans le Sud de la région betsileo sous l'impulsion d'un devin-guérisseur repenti et converti au protestantisme, Rainisoalambo, le fifohazana est un mouvement de Réveil protestant, néanmoins intégré à l'Église luthérienne malgache (FLM). II place les soins par l'exorcisme au centre des activités de ses membres. Les exorcistes laïcs du mou-
} 
lien entre la présence d'un ethnologue et leur pratique religieuse. À ce titre, ils assurent que Jésus Christ m’a poussée à choisir cet objet d'étude. Ils poursuivent par une comparaison avec leur propre appel divin. En effet, au début de leur expérience avec le mouvement de Réveil, les futurs exorcistes n'ont pas conscience ni de leur future conversion au protestantisme, ni de leur future ordination. Ils m'inscrivent dans ce cheminement et me positionne à l'étape initiale du processus, celui de la méconnaissance du phénomène divin (moment qui précède le refus de l'appel, lui-même suivi de son acceptation). Cet aspect est un des invariants des conditions d'accès à la fonction d'exorciste : l'appelé est inconscient avant de finalement comprendre le message divin que Jésus Christ lui envoie. Comme le souligne de nombreux exorcistes lors du discours tenu sur le récit de l'apparition de leur vocation, une de mes interlocutrices mentionne les nombreux appels qu'elle a laissé sans réponse (cet invariant de l'appel divin ou de l'apparition d'un don se retrouve dans de nombreuses guérisons religieuses malgaches : possédés, devins-guérisseurs ou exorcistes). Cette dernière a ensuite interprété le message divin comme suit : « je ne m'en rendais pas encore compte. Et à ce moment-là, j'ai été vraiment convaincue : je vais être mpiandry ${ }^{7}$ ». Autrement dit, mon tour viendra. Devenir exorciste implique un changement radical de mode de vie, alors jugé diabolique. À leur prise de fonction, les guérisseurs protestants exhortent fréquemment l'arrêt de toute consommation d'alcool, de tabacs ou la pratique de certaines conduites sexuelles. Les nouveaux exorcistes confortent la réception de l'appel divin par un reniement et un remaniement de leur vie passée. Dès lors, ils mettent en avant ces nouvelles "valeurs morales que leur adhésion au mouvement avaient introduites dans leur vie quotidienne » (Mary, 2000).

Ainsi, les membres du mouvement de Réveil diabolisent les pécheurs, parmi lesquels, les devins-guérisseurs. Les exorcistes les mentionnent dans leur rapport aux esprits, et particulièrement dans les cas d'alliances avec les filles-des-eaux, y voyant des pratiques sexuelles déviantes. Les exorcistes préconisent le recours au mouvement du fifohazana comme unique garantie de guérison face aux démons présents en masse dans la société dite «moderne ». Ce discours est possible via une «interprétation dramatisante » (Rakotomalala et al., 2001:123) des souffrances sociales, familiales ou nationales véhiculées par les médias, mais aussi certaines conduites sexuelles (adultères, homosexualité, libertinage sexuel, etc.), certains courants musicaux, les jeux vidéo, la mondialisation, les films pornographiques, etc.

Par exemple, dans un des centres d'accueil des malades (toby) du mouvement de Réveil protestant de Fianarantsoa vit une jeune femme arrivée dans la structure en 2009. L'exorciste laïc (mpiandry) du mouvement qui la prend en charge remarque que

vement pratiquent ainsi la chasse des démons et l'imposition des mains dans les temples, à domicile ou dans les centres d'accueil des malades gérés par le fifohazana.

7 Traduction d'un extrait d'entretien réalisé le 11 septembre 2011 à Antamponjina, Fianarantsoa. 
ses conduites sexuelles volages sont la raison pour laquelle les démons ont été attirés en elle: « elle était une fille pas sage qui suit les voitures [sous-entendu "qui monte dans les voitures de jeunes hommes inconnus pour avoir des relations sexuelles avec eux"], quand elle était jeune. Et c'est à partir de ça qu'elle a eu des devoly [“démons"] Dans le contexte évangélique suisse, il est également remarqué que «les pratiques sexuelles déviantes [ou considérées comme telles] confirment l'influence démoniaque dans le registre des corps" (Gonzalez, 2008: 49). Comme avec l'exemple de l'expression «suivre les voitures », les conduites sexuelles sont sous-entendues dans le discours mais permettent néanmoins d'évoquer le contexte religieux, d'en justifier les pratiques, ou au contraire, de les brimer.

\section{Discours tenus sur la sexualité}

\subsection{Représentations et imaginaire de la figure de la sorcière}

Les devins-guérisseurs, praticiens du culte aux esprits, sont des individus précis et reconnus par la société, pouvant être désignés par pas moins de huit termes en malgache. Ce phénomène peut être remarqué tant en théorie qu'en pratique. D'ailleurs, pour la majorité de mes interlocuteurs, une partie de ces huit termes se valent malgré leurs nuances, excepté celui de mpamosavy ("sorcier»). Il désigne celui qui jette les sorts, mais ne les enlève pas. Il demeure très discret, officiellement, personne ne dit connaître de mpamosavy.

Le ou la mpamosavy est celui qui fait des mosavy, des mauvais sorts. D'autres termes ont également été répertoriés : ceux, dont le sens est atténué, ody rasty ou fanafody rasty (" mauvais charme» et «mauvais remède »), celui renvoyant à la puissance du sorcier, ody mahery (« charme fort») et enfin le terme de vorika, synonyme de mosavy qui compte parmi les plus régulièrement utilisés par mes interlocuteurs avec celui de mosavy. Dans le contexte malgache, le désordre social est l'affaire du mpamosavy, vecteur des conséquences des fautes des uns et des jalousies des autres. La figure du sorcier est construite à l'opposé de la " parenté étendue du fihavanana » (Mouzard, Gueunier, 2009: 302), l'idéal social de la parenté élargie.

La mpamosavy, figure généralement féminine, se déplace et opère, d'après les représentations populaires, le plus souvent de nuit et accompagnée de près ou de loin par des chats sauvages (kary) ou des hiboux (vorondolo). « Les chats sont censés fréquenter les tombeaux et avoir une affinité avec le monde des morts et le domaine de la sorcellerie » (Beaujard, 2009: 262). Les sorcières sont généralement décrites avec une chevelure en bataille et vêtues de guenilles ou intégralement nues. Dans le contexte très pudique de la société malgache, la nudité sous-entend la folie et l'avidité sexuelle. En

${ }^{8}$ Traduction d'un extrait d'entretien réalisé le 6 mai 2010 à Soatsihadino, Fianarantsoa. 
opposant ainsi les mpamosavy à tout être doté de raison morale et sociale, elles sont ainsi considérées comme des êtres humains hors normes. L'évocation de la nudité définit alors aussi bien une sorcière qu'une folle. Ainsi, «hommes et femmes portent leur lamba ["étoffe"] craignant d'être taxée de manary lamba (jeter son lamba), se montrer nu, ce qui signifie fou ou folle à lier car ne connaissant plus la honte. Le lamba est synonyme de sagesse, de respect et de dignité humaine » (Razafiarivony, 2000 : 425426), soit une anti-définition du mpamosavy. Les personnes décrétées folles ou sorcières sont bannies du tombeau familial. Leur dépouille repose dans une tombe annexe. Les sorciers ou, plus précisément, les sorcières sont évoqués rôdant autour des tombeaux à la nuit tombée, chantant dans les cours des maisons pour attirer les hommes (bien que tous les entendent) qu'elles ont choisis pour la nuit. L'homme, hypnotiquement attiré hors de son domicile, se place à quatre pattes et sera chevauché par la mpamosavy, puis il aura, éventuellement, des relations sexuelles avec elle pour ensuite regagner sa maison avant le lever du soleil.

À propos des représentations des sorcières, un de mes interlocuteurs, par ailleurs exorciste du mouvement de Réveil, raconte qu'il y a plusieurs années, devant se rendre à Tananarive à plus de quatre cents kilomètres au nord de Fianarantsoa, il entreprit un voyage de nuit. Seul en voiture, en rase campagne, il aperçut au détour d'un virage une femme éclairée par ses phares. Le doute et la peur s'installent alors en lui, car il est dit que «la nuit appartient aux sorciers », qui sont aussi appelées mpivoaka alina: «ceux qui ont l'habitude de sortir la nuit » (Rakotomalala, 2006:243). Il freine et voit une femme nue, qu'il identifie immédiatement comme une sorcière errant dans la nuit. Au début du XX $\mathrm{X}^{\mathrm{e}}$ siècle, Henri Dubois (1938) décrivait les sorciers, les appelant aussi les mpandeha alina (« coureurs de nuit») : " on les voit, le corps entièrement nu » (p. 909), poursuit l'auteur. Dès lors, le voyageur s'est immédiatement enfermé dans sa voiture craignant de croiser le regard de cette femme qui pourrait l'attirer et l'entraîner vers un tombeau, dit-il, sous-entendant à la fois l'appréhension d'une entreprise magico-sorcellaire et la crainte d'une emprise sexuelle. Finalement, il put arriver à Tananarive, en évitant les contacts visuels et charnels avec la supposée sorcière. Mais cette rencontre lui a laissé un amer souvenir qui l'a amené à prendre la décision de ne plus jamais voyager de nuit par appréhension d'une nouvelle rencontre avec une « coureuse de nuit » dénudée.

\subsection{Mise à distance corporelle}

Comme les sorcières, les maux sexuels sont dans le cas ethnographique précédent soigneusement contournés par certains guérisseurs. Si RaJean-Pierre mentionne avec le plus grand sérieux ses rapports aux entités aquatiques et à leur féminité exacerbée, il tourne en dérision les préoccupations thérapeutiques d'un malade localisant à demi-mot sa douleur au niveau du fessier. Entre le discours magico-religieux de son iti- 
néraire initiatique et la réalité des maux de ses patients, RaJean-Pierre montre des formes discursives variées.

Le patient, un homme d'une soixantaine d'années, expose ses symptômes. RaJeanPierre commence ses préparations et entame les soins. Il extrait un sisika du sommet du crâne de l'homme qui souffre de céphalées. Les sisika sont des attaques sorcellaires placées sous la peau de la victime sous la forme de bouts de bois provenant d'un cercueil ou d'une planche de transport mortuaire. Leur extraction spectaculaire, par une technique d'aspiration buccale, est une des spécialités thérapeutiques de RaJeanPierre. Le devin-guérisseur pense avoir fini les traitements, lorsque le malade chuchote: «j'ai mal là, aussi ${ }^{9}$ », désignant son fessier. La réaction de Rajean-Pierre est immédiate et non-négociable, il secoue la tête et s'exclame : «Hohoho!». Son épouse, qui l'assiste, essaye de le convaincre mais il rétorque : « je ne peux pas aller voir si c'est là ! [Rires] $]^{10}$.» Ce refus de RaJean-Pierre de retirer des sisika de certaines parties du corps renvoie à la " hiérarchisation des différentes parties du corps, réprimant toute inversion et confusion des parties, combinant inversion et confusion avec la notion de souillure [...] (inversion tête/fesses; souillure des excréments) 》 (Rakotomalala, 2006: 251). Rajean-Pierre finit par souligner, d'un ton sérieux, que le sisika déjà enlevé ce jour, ajouté à ceux extraits lors des consultations précédentes, devrait aboutir à la nette amélioration de la santé de ce malade.

Si le contact direct, et plus encore buccal, avec des zones du corps considérés comme sexualisées est un problème pour Rajean-Pierre, l'évocation de la sexualité n'est pas rare pour autant dans les salles de soins. Ainsi de nombreux guérisseurs ont insisté, lors des temps d'entretien, sur les symboliques masculine ou féminine des plantes médicinales et des perles magico-religieuses ${ }^{11}$ : ces éléments rituels évoquent, symboliquement et dans le discours à visée pédagogique des devins-guérisseurs, le masculin lorsqu'ils sont de forme allongée et le féminin lorsqu'il présente une forme ovoïde.

\subsection{Humour potache et demandes explicites}

Alors que les patients sont discrets sur les questions de sexualité face aux devinsguérisseurs, les esprits qui possèdent ces derniers, quant à eux, sont assez loquaces sur ce point. C'est le cas de l'esprit de Rakotomaditra. Il se présente comme un militaire (voire aussi Rakotomalala et al., 2001) et est aussi défini ainsi par une jeune possédée de Fianarantsoa, en dehors des séances de possession. Lors de sa présentation

${ }_{9}^{9}$ Traduction de propos recueillis lors d'une consultation, le 6 juin 2011 à Ambalavato, Fianarantsoa.

${ }^{10}$ Idem.

${ }^{11}$ Rakotomalala (2012) a observé une symbolique semblable appliquée aux pierres rituelles au nord des Hautes Terres. 
rituelle, l'esprit raconte: "avec les autres militaires, on buvait et on fumait du chanvre [rongony ${ }^{12}$." Rakotomaditra est un esprit réputé dans le paysage religieux malgache, fils de la célèbre Ranoro (zanahary, vazimba ou esprit aquatique selon les mythes) à qui un lieu de culte réputé est dédié près de Tananarive. Le caractère de Rakotomaditra fait également sa renommée, en premier lieu par son nom: maditra évoque l'idée d'un voyou, d'un délinquant, d'un rebelle (Blanchy, 2010), d'un enfant terrible et intenable (Rakotomalala et al., 2001). Il est aussi présenté comme ayant un fort penchant pour l'alcool. Rakotomaditra, qui possède la jeune guérisseuse, plaisante avec un malade lors d'un rituel contre-sorcellaire : « mets ton œil dedans [l'eau sacralisée] ! [Rires $]^{13} »$, puis il affirme qu'il va s'occuper de la femme qui l'a rendu malade (celle à l'origine de l'attaque sorcellaire), ce qu'il exprime vulgairement avec des allusions sexuelles, tandis qu'il éteint un bâtonnet d'encens en le plongeant dans le bol contenant l'eau sacralisée.

D’autres devins-guérisseurs, quant à eux, proposent à leurs patients masculins des allongements ou des élargissements de pénis par des massages et des infusions comme l'a mis en avant Malanjaona Rakotomalala à partir de données fournies par Delphine Burguet (2012). Certaines prescriptions mentionnent uniquement des infusions (tambavy) après que les patients soient venus consulter avec un ou plusieurs morceaux de bois symbolisant la taille et/ou le diamètre du sexe qu'ils souhaitaient obtenir, selon mes observations sur le lieu de culte forestier de Donné, dans les environs de Fianarantsoa. Il est alors particulièrement intéressant de noter que ces demandes, on ne peut plus intimes et formulées explicitement, ont été collectées par deux ethnographes femmes et non-malgaches, alors que les discours sur la sexualité sont souvent pudiquement tus. Il est envisageable de percevoir ce point comme un aspect «technique » ne présentant pas de gêne à être divulgué à des oreilles à la fois profanes et étrangères car il n'évoque pas la relation de couple, identifiant alors précisément la compagne du patient. Il est aussi possible d'y voir un aspect plus méthodologique : en effet, si un devin-guérisseur accorde sa confiance à un ethnologue, ses patients feront de même, y compris dans des cas concernant la sexualité, la fertilité, etc. Lors des consultations visant à traiter la stérilité d'un couple, ce problème est toujours présenté comme étant un mal imputé à la femme. Si la stérilité est féminine, les raisons de la naissance d'un couple et ses rapports intimes ne sont pas genrés, y compris en cas d'intervention magico-sorcellaire.

\footnotetext{
12 Propos recueillis le 30 mai 2013 à Ambodirano, Fianarantsoa.

${ }^{13} \mathrm{ldem}$.
} 


\section{Silence sur la sexualité et bavardage sur les philtres d'amour}

Le silence entourant les rapports de couple et la sexualité dans les temps d'enquête est à penser en parallèle des temps longs voués aux discours sur l'usage, la confection, les spécialistes, les usagers et les victimes des philtres d'amours, dans et hors des salles de soin des devins-guérisseurs. Les ody fitia ("charmes d'amour») ne sont-ils qu'une « forme bénigne » (Ottino, 1998 : 462) de la sorcellerie?

Les spécialistes eux-mêmes ne sont pas catégoriques sur ce point et remarquent le caractère flou des frontières entre les différents termes désignant les devinsguérisseurs, tout comme certains perçoivent les philtres d'amour d'un côté ou de l'autre de la frontière de la sorcellerie. Malanjaona Rakotomalala (2006) place les philtres d'amour ou « mosavy [acte sorcellaire] des relations amoureuses », utilisés par les jeunes filles de Tananarive visant à « retenir leurs amants ", dans la catégorie de la sorcellerie (p. 237-238). Un des charmes de contraintes le plus répandu dans les demandes exposées aux devins-guérisseurs, est l'ody fitia (" charme d'amour »).

RaGilles est le seul de mes interlocuteurs à avoir explicitement évoqué des pratiques sorcellaires. Il introduit cette activité en narrant sa vie conjugale. RaGilles a été veuf deux fois et s'est marié trois fois. À la vue de ses jeunes et nombreuses épouses, la rumeur a vite gagné le quartier du centre de Fianarantsoa où il réside et pratique ses activités. La rumeur n'est que plus persistante du fait des particularités de RaGilles. Né en 1937, dans une famille protestante qu'il présente comme très croyante, RaGilles avait également une ancêtre paternelle guérisseuse : "la grand-mère de ma grandmère faisait ces trucs-là ${ }^{14}$. » Cette aïeule originaire de la région de Vohipeno dans le quart sud-est de l'île, éminemment réputée pour la puissance de ses guérisseurs et sorciers, accentue encore la réputation de RaGilles, issu d'autre part, d'une famille betsileo. Il souffre de déformations des mains et des pieds l'empêchant de se mouvoir et lui créant des difficultés de préhension. Il est également, en plus de ses activités de guérisseur, catéchiste de la paroisse protestante luthérienne voisine. RaGilles, malgré ses handicaps et sa position sociale complexe, a eu trois épouses reconnues comme jeunes et belles par la rumeur locale. Son épouse actuelle, une exorciste protestante, mpiandry, d'une trentaine d'années surenchérit: «il peut avoir des jolies femmes ! $\mathrm{Ha}$ ! Il sait très bien faire ${ }^{15}$ !» Il mobilise alors ces aspects de sa vie intime pour prouver l'efficacité de ses philtres d'amour : «je fais les ody fitia, c'est ça qui intéressent les filles et les garçons ! C'est ça que j'utilise ici ${ }^{16}$. » Cependant, RaGilles n'arrête pas là son discours sur l'utilisation des charmes sorcellaires ; il justifie sa pratique en se référant à la Bible qu'il a toujours à portée de main. RaGilles cite le verset 2.24 de la Genèse : « c'est pourquoi l'homme quittera son père et sa mère, et s'attachera à sa femme, et ils

${ }^{14}$ Traduction d'un extrait d'entretien réalisé le 4 mai 2010 à Ampitakely, Fianarantsoa.

15 Idem.

${ }^{16}$ Idem. 
deviendront une seule chair ${ }^{17}$.» La fierté de ses réussites conjugales l'amène à poursuivre en proposant ses services en la matière au jeune homme qui me l'a présenté, tout en me regardant en souriant. RaGilles intercède ainsi comme spécialiste compétent en la matière. Le discours sérieux dont il fait montre lorsqu'il mentionne la chrétienté et ses aïeux puissants se transforme en sourire et œillade entendue lorsqu'il évoque les ody fitia et leur issue sexualisée. Ces charmes ont de nombreux effets: «rendre amoureux ou provoquer une véritable passion, séparer un couple, attirer l'autre pour satisfaire un besoin sexuel, le rendre fou, tuer la personne si elle en préfère une autre », etc. (Rakotomalala, 2012 : 258), tous présupposés ou énoncés par RaGilles lorsqu'il dresse une liste non-exhaustive de ses compétences en la matière.

\section{Conclusion}

La sexualité, qu'elle soit la conséquence d'une attaque sorcellaire, la finalité d'une alliance avec un esprit, l'aboutissement d'une technique d'agrandissement du pénis ou qu'elle soit mythifiée et fantasmée comme pour les sorcières avides de rencontres nocturnes ou les filles-des-eaux attirants les hommes dans leurs filets, est bien présente dans les discours magico-religieux populaires et dans ceux des officiants du culte. Ces derniers se réfèrent essentiellement à des expériences personnelles (initiatique ou cultuelle), tout en mettant à distance l'acception conjugale des conduites sexuelles. Autrement dit, la sexualité est, pour cet exemple ethnographique, particulièrement évoquée dans son lien à la religiosité. Elle est façonnée par les religiosités en présence (cultes aux esprits et protestantisme pour les cas mentionnés ici) dans les préoccupations quotidiennes, les rumeurs ou les remarques destinées à l'ethnologue.

Les éléments mentionnés ici ont donc permis d'élaborer une première réflexion sur la place de la sexualité dans les discours et les pratiques magico-religieuses. En ce sens, ils ont également ouvert à des ébauches de questionnements quant aux rapports qu'entretiennent divers guérisseurs à la sexualité dans un contexte religieux, qu'ils aient énoncé explicitement ces éléments ou qu'ils les aient sous-entendus face à un auditoire profane fait de patients, de croyants ou d'une ethnologue.

\section{Bibliographie}

BEAUJARD P. (2009), « La place et les pratiques des devins-guérisseurs dans le Sud-Est de Madagascar ", in D. NATIVEL et F. V. RAJAONAH, Madagascar revisitée. En voyage avec Françoise Raison-Jourde, Paris, Karthala, p. 259-286.

\footnotetext{
${ }^{17}$ Ary noho izany ny lehilahy dia handao ny rainy sy ny reniny ka hikambana amin'ny vadiny; dia ho nofo iray ihany ireo. Genesisy 2.24.
} 
Blanchy S. (2010), «Vierge, Mère ou Reine ? », Archives de sciences sociales des religions, $n^{\circ} 150$, p. 135-154.

BuRGUET D. (2012), « Le jeune guérisseur et la sainte kalanoro, l'innovation rituelle du culte aux esprits de la nature », Journal des anthropologues, $\mathrm{n}^{\circ} 128-129$, p. 189-208.

De FlaCourt É. (2007 [1658]), Histoire de la grande isle Madagascar, Paris, Karthala.

Descola P. (1993), Les lances du crépuscule. Relations Jivaros, haute Amazonie, Paris, Plon (« Terre humaine »).

Dubois H. (1938), Monographie des Betsileo, Paris, Institut d'ethnologie.

FIELOUX M., LOMBARD J. (1991), Le prince charmant, film documentaire, IRD/MRSTD Madagascar, édition CERIMES (Centre de ressources et d'information sur les multimédias pour l'enseignement supérieur), $43 \mathrm{~min}$.

GoNZALEZ P. (2008), «Lutter contre l'emprise démoniaque. Les politiques du combat spirituel évangélique », Terrain, $\mathrm{n}^{\circ}$ 50, p. 44-61.

HAMAYON R. (1990), La chasse à l'âme. Esquisse d'une théorie du chamanisme sibérien, Nanterre, Société d'ethnologie.

HEBERT J-C. (1964), «Filan’ Ampela, ou propos galants des Sakalava », Journal de la Société des africanistes, vol. 34, $\mathrm{n}^{\circ}$ 2, p. 227-254.

MARY A. (2000), "Conversion et conversation. Les paradoxes de l'entreprise missionnaire », Cahiers d'études africaines, $\mathrm{n}^{\circ} 160$, p. 779-800.

MouzARD T., GueUnier N. J. (2009), « Récits de séjours non-éternels. La mort fugitive comme voie d'accès à la médiation à Madagascar (possession, prédication)», in D. Nativel, F. V. RajaOnah (dir.), Madagascar revisitée. En voyage avec Françoise Raison-Jourde, Paris, Karthala, p. 287-352.

Otтino P. (1998), Les champs de l'ancestralité à Madagascar. Parenté, alliance et patrimoine, Paris, Karthala, ORSTOM.

Rakotomalala M. (2006), "La sorcellerie en Imerina », in S. Blanchy, J. A. RAKOtOARISOA, P. BeAUjARD, C. RADimilahy (dir.), Les dieux au service du peuple. Itinéraires religieux, médiations syncrétisme à Madagascar, Paris, Karthala (« Religions contemporaines »), p. 229-260.

Rakotomalala M. (2010a), « Présentation », Études océan Indien, n 45, p. 7-12.

Rakotomalala M. (2010b), « Mots et expressions merina sur la sexualité (Hautes Terres centrales de Madagascar) », Études océan Indien, n 45, p. 149-231.

Rakotomalala M. (2012), À cœur ouvert sur la sexualité merina (Madagascar). Une anthropologie du non-dit, Paris, Karthala (« Hommes et sociétés »). 
Rakotomalala M., Blanchy S., Raison-Jourde F. (dir.) (2001), Madagascar : les ancêtres au quotidien. Usages sociaux du religieux sur les Hautes-Terres malgaches, Paris, L'Harmattan.

RAZAFIARIVONY M. (2000), "Le "lamba": une expression de l'identité malgache », in C. Allibert, N. RAJAONARIMANANA (dir.), L'extraordinaire et le quotidien. Variations anthropologiques, Paris, Karthala, p. 423-434.

SALAdiN D’ANGLURE B., MORIN F. (1998), " Mariage mystique et pouvoir chamanique chez les Shipibo d'Amazonie péruvienne et les Inuit du Nunavut canadien», Anthropologie et Sociétés, vol. 22, $\mathrm{n}^{\circ}$ 2, p. 49-74.

Terramorsi B. (2010), "La femme-poisson ou l'apnée du sommeil de la raison », in B. Terramorsi (dir.), Les filles des eaux dans l'océan Indien. Mythes, récits, représentations, Paris, L’Harmattan, p. 9-22. 